\title{
Accuracy of emergency department triage using the Emergency Severity Index and independent predictors of under-triage and over-triage in Brazil: a retrospective cohort analysis
}

\author{
Jeremiah S. Hinson ${ }^{1 *}$, Diego A. Martinez ${ }^{1,2}$, Paulo S. K. Schmitz ${ }^{3}$, Matthew Toerper ${ }^{1,2}$, Danieli Radu ${ }^{3}$,
} James Scheulen ${ }^{1}$, Sarah A. Stewart de Ramirez ${ }^{1 \dagger}$ and Scott Levin ${ }^{1,2,4,5+}$

\begin{abstract}
Background: Emergency department (ED) triage is performed to prioritize care for patients with critical and time-sensitive illness. Triage errors create opportunity for increased morbidity and mortality. Here, we sought to measure the frequency of under- and over-triage of patients by nurses using the Emergency Severity Index (ESI) in Brazil and to identify factors independently associated with each.

Methods: This was a single-center retrospective cohort study. The accuracy of initial ESI score assignment was determined by comparison with a score entered at the close of each ED encounter by treating physicians with full knowledge of actual resource utilization, disposition, and acute outcomes. Chi-square analysis was used to validate this surrogate gold standard, via comparison of associations with disposition and clinical outcomes. Independent predictors of under- and over-triage were identified by multivariate logistic regression.

Results: Initial ESI-determined triage score was classified as inaccurate for 16,426 of 96,071 patient encounters. Undertriage was associated with a significantly higher rate of admission and critical outcome, while over-triage was associated with a lower rate of both. A number of factors identifiable at time of presentation including advanced age, bradycardia, tachycardia, hypoxia, hyperthermia, and several specific chief complaints (i.e., neurologic complaints, chest pain, shortness of breath) were identified as independent predictors of under-triage, while other chief complaints (i.e., hypertension and allergic complaints) were independent predictors of over-triage.
\end{abstract}

Conclusions: Despite rigorous and ongoing training of ESI users, a large number of patients in this cohort were under- or over-triaged. Advanced age, vital sign derangements, and specific chief complaints-all subject to limited guidance by the ESI algorithm—were particularly under-appreciated.

Keywords: Triage, Mistriage, Emergency severity index, Emergency department

\footnotetext{
* Correspondence: hinson@jhmi.edu

tEqual contributors

${ }^{1}$ Department of Emergency Medicine, Johns Hopkins University School of

Medicine, 801 Smith Avenue, Davis Building, Suite 3220, Baltimore, MD

21209, USA

Full list of author information is available at the end of the article
} 


\section{Background}

The primary objective of triage is to rapidly identify patients with critical and time-sensitive conditions and to prioritize their care above those who can wait [1]. Effective triage is required when demand for medical care outstrips capacity, as has become commonplace in the emergency department (ED) due to overcrowding, now recognized as a major threat to patient safety and quality care across the globe [2-5].

In this context, the accuracy and reliability of ED triage are paramount. Under-triage, or failure to identify and differentiate patients with acutely severe illness (e.g., myocardial ischemia, sepsis) from those with less urgent needs (e.g., indigestion, minor infections), contributes to delays in time-sensitive interventions and to potentially avoidable clinical deterioration, morbidity, and mortality [6-9]. Consequential delays to thrombolytic therapy [10], percutaneous coronary intervention [11], antibiotic administration [12], asthma treatment [13], and analgesic administration [14] have all been associated with ED crowding and place under triaged patients at undue risk. Over-triage, or inappropriate labeling of patients with non-urgent presentations to high acuity designations, may have indirect, but equally harmful effects. Triage level designation is often associated with tracking of patients to specific ED care locations based on anticipated resource need [15], and triage level has been shown to drive physician resource utilization decisions, including hospital admission [16]. Thus, over-triage results in diversion of limited time and resources from patients most in need and inappropriate allocation to those with less severe illness.

The most widely used ED triage tools employ a fivelevel triage scale and include the Australian Triage Scale [17], Canadian Emergency Department Triage and Acuity Scale (CTAS) [18], Manchester Triage Scale (MTS) [19], and the Emergency Severity Index (ESI) [20]. ESI was developed in the USA and is being adopted by an increasing number of EDs globally [20, 21]. Despite its widespread adoption and numerous strengths that include ease of use and linkage to anticipated ED resource utilization [20], ESI has several limitations. It relies heavily on provider judgment and intuition, allowing for significant practice variation, with inter-rater reliability reported to range from $k=0.46$ to 0.91 [22]. More than half of all visits in the USA are triaged to ESI level 3, generating a large pool of undifferentiated patients that creates challenges for efficient ED resource distribution and effective patient queuing, undermining the very purpose of triage [23, 24] Furthermore, ESI has never been well-validated against critical outcomes indicating time-sensitive needs in any setting [20].

In this study, we evaluated the performance of ESI in the ED of a large tertiary care academic hospital in Porto
Alegre, Brazil, and identified independent predictors of under- and over-triage by nurses using ESI. We also measured the impact of under- and over-triage on hospitalization and critical outcomes.

\section{Methods \\ Study design}

We used a single-center retrospective cohort study design to characterize ED triage patterns. Records for all ED visits during the study period were retrieved from a relational database underlying the study institution electronic health record (EHR) by an experienced data user and were de-identified prior to analysis by the study team. This study was approved by the institutional review boards of all participating institutions.

\section{Study setting and population}

All patient visits occurred at a large tertiary care academic hospital in Porto Alegre, Brazil, with Joint Commission International accreditation, an ED census of 78,000 visits per year and a mean annual admission rate of $12 \%$. All ED triage at this institution is performed using ESI, and all study site triage nurses have undergone formal training in the use of ESI, passed ESI competency exams, and receive annual refresher training sessions. Visits for adult patients ( $\geq 18$ years old) who presented for care between January 1, 2013, and September 13, 2015, were included for analysis.

\section{Measures}

Triage level designations and gold standard comparator

As a part of routine clinical care, ESI triage levels were assigned by a nurse with formal training in ESI for all patients at the time of ED arrival. Nurse-assigned ESI triage level was used to guide clinical care. For administrative purposes unrelated to this study, a second ESI triage level was entered for all patients at the close of ED encounter by the treating emergency physician. Both nurse and physician triage level designations were made according to the standardized ESI algorithm, but physician ESI level designation was made with full knowledge of actual ED resource utilization and acute clinical outcomes. Physician-assigned ESI triage level (assigned a posteriori) was used as a surrogate gold standard for accurate triage and was validated as such by the measurement of association with hospital admission and composite critical outcome using chi-square analysis (see Fig. 2). Prior to analysis, ESI triage scores were designated as high acuity (ESI level 1 or 2), moderate acuity (ESI level 3), or low acuity (ESI level 4 or 5). Redistribution of triage scores from five to three tiers was performed prior to analysis to more effectively capture the clinical impact of triage decisions, as ESI triage levels 1 and 2 are considered time-sensitive and are roomed 
immediately, while ESI level 3 patients often wait hours to receive definitive care, and ESI levels 4 and 5 are cared for in a separate area of the ED with a fast track designation. Thus, under-triage from ESI level 4 to ESI level 5 (both low acuity and subject to similar clinical care pathways) would be expected to have a much smaller effect on patient care and outcomes than under-triage from ESI level 3 (moderate acuity) to ESI level 4 (low acuity), and redistribution to three tiers (high, moderate, low) allowed us to account for this differential effect.

\section{Definitions of under- and over-triage}

Any patient assigned to a triage tier of lower acuity on arrival (nurse-assigned) than at close of encounter (physician-assigned) was defined as under-triaged. Any patient assigned to a triage tier of higher acuity on arrival than at close of ED encounter was defined as overtriaged. Patients with the same designation on arrival and at close of ED encounter were defined as accurate.

\section{Clinical outcomes}

Clinical outcome measures included hospital admission, in-hospital mortality, and multiple critical patient outcomes. Hospital admission was defined as any admission to an inpatient care site. In-hospital mortality was defined as death during the index hospital encounter, irrespective of whether it occurred in the ED or during inpatient hospitalization. Composite critical outcome was defined as meeting one or more of the following criteria within $24 \mathrm{~h}$ of ED disposition: admission to an intensive care unit (ICU), emergent procedure in an operating room, cardiac catheterization, endoscopy or bronchoscopy, transfer to an outside acute care facility, or in-hospital mortality.

\section{Candidate predictor variables}

Patient data collected at the time of ED triage and used as candidate predictor variables for under- and overtriage by multivariate logistic regression analysis (see below) included age, sex, vital signs, patient-reported pain score, chief complaint category, and arrival time. Vital signs, including temperature, systolic blood pressure, heart rate, respiratory rate, and oxygen saturation, were classified as normal or gradations of abnormal according to previously established physiologic cutoffs [25-28]. Pain scores were obtained using a visual analog scale that ranged from 1 (least severe) to 10 (most severe), and scores were further classified as mild $(\leq 3)$, moderate $(4-7)$, or severe $(\geq 8)$. Free-text chief complaints were extracted directly from the electronic health record (EHR), and natural language processing software (Python 2.7 with Natural Language Toolkit 3.0) was used to map complaints to 1 of 30 chief complaint categories derived from a schema developed by the Agency for Healthcare Research in Quality as previously described [24, 29].

\section{Data analysis}

Dichotomous and categorical data were displayed as absolute and relative frequencies (in percentages) and continuous data as medians with interquartile ranges (IQRs). Rates of under- and over-triage were calculated as the percentage of visits assigned to a higher or lower triage level on arrival than at close of encounter, respectively. Missing data (i.e., sex, vital signs, pain score, chief complaint) were recorded as null and included in logistic regression analysis (below). Chi-square analysis: Associations between under-triage or over-triage and hospital admission or composite critical outcome were assessed using Pearson's chi-squared test with Yates' continuity correction. Multivariate Logistic Regression Analysis: Primary outcome measures included rates of under-triage, over-triage, and concordance (accurate triage). We investigated the extent to which specific patient characteristics (age, sex, temperature, pulse, respiratory rate, systolic pressure, oxygen saturation, patientreported pain score, chief complaint category, and arrival time) were associated with triage score concordance. Logistic regression models of triage misclassification (over- or under-triage versus concordance) that simultaneously incorporated the several patient characteristics were generated. All analyses were performed in $\mathrm{R}$ version 2.14.1 using freely distributed statistical packages.

\section{Results}

A total of 96,071 unique adult patient visits were included in our analysis. Patient demographics and clinical characteristics are shown in Table 1. Nearly half of all patient visits (49.1\%) were triaged as moderate acuity (ESI level 3) on ED arrival. Roughly one third (32.5\%) were triaged as low acuity (ESI level 4 or 5), and only $18.3 \%$ were triaged as high acuity (ESI level 1 or 2) (Fig. 1). Using a posteriori physician-assigned triage levels as surrogate gold standard comparators, one fifth of all patients were classified as either under- or overtriaged. For patients triaged to high acuity ESI levels, $8.7 \%$ were classified as over-triaged. For patients triaged to moderate acuity on arrival, $13.6 \%$ were classified as over-triaged and $5.8 \%$ were classified as under-triaged. $18.4 \%$ of patients assigned to low acuity ESI levels were classified as under-triaged (Fig. 1).

\section{Under-triage and risk for admission or critical outcome}

As demonstrated in Fig. 2a, patients who were triaged as moderate acuity by ESI (level 3) on arrival but subsequently determined to be more appropriate for high acuity triage level 1 or 2 (i.e., under-triaged) had a 
Table 1 Patient demographics and clinical characteristics

\begin{tabular}{|c|c|}
\hline Age (IQR) & $39(29.7-59.2)$ \\
\hline \multicolumn{2}{|l|}{$\operatorname{Sex}(\%)$} \\
\hline Female & $59,091(61.51)$ \\
\hline Male & $36,632(38.13)$ \\
\hline \multicolumn{2}{|l|}{ Vitals (IQR) } \\
\hline Temperature (Fahrenheit) & $97.3(96.6-98.2)$ \\
\hline Pulse (bpm) & $85(75.0-98.0)$ \\
\hline Respiratory rate (rpm) & $20(18.0-20.0)$ \\
\hline Systolic blood pressure $(\mathrm{mmHg})$ & $128(117.0-141$. \\
\hline Oxygen saturation (\%) & $98(97.0-99.0)$ \\
\hline \multicolumn{2}{|l|}{ Triage level (\%) } \\
\hline ESI 1 & $272(0.28)$ \\
\hline ESI 2 & $17,334(18.04)$ \\
\hline ESI 3 & $47,207(49.14)$ \\
\hline ESI 4 & $29,921(31.14)$ \\
\hline ESI 5 & $1337(1.39)$ \\
\hline \multicolumn{2}{|l|}{ Arrival time (\%) } \\
\hline 8 AM-12:59 PM & $30,565(31.82)$ \\
\hline 1 PM-4:59 PM & $23,085(24.03)$ \\
\hline 5 PM-8:59 PM & $19,620(20.42)$ \\
\hline 9 PM-12:59 AM & $12,941(13.47)$ \\
\hline 1 AM-7:59 AM & $9860(10.26)$ \\
\hline \multicolumn{2}{|l|}{ VAS pain score (\%) } \\
\hline Mild (0-3) & $39,376(40.99)$ \\
\hline Moderate [4-7] & $32,517(33.85)$ \\
\hline Severe $[8-10]$ & $22,836(23.77)$ \\
\hline Hospital admission (\%) & $14,508(15.10)$ \\
\hline \multicolumn{2}{|l|}{ Critical outcomes (\%) } \\
\hline ICU admission & $183(0.19)$ \\
\hline Death & 1019 (1.06) \\
\hline Surgery & $1991(2.07)$ \\
\hline Cardiac catheterization & $219(0.23)$ \\
\hline Hospital transfer & $48(0.05)$ \\
\hline In-hospital mortality & $1290(1.34)$ \\
\hline
\end{tabular}

bpm beats per minute, ICU intensive care unit, IQR interquartile range, rpm respirations per minute, VAS visual analog scale

significantly increased prevalence of admission and critical outcomes as compared to those appropriately triaged to moderate acuity $\left(\chi^{2}=502.06, \mathrm{df}=1, p\right.$ value $<0.001$ and $X^{2}=184.91, \quad \mathrm{df}=1, \quad p$ value $<0.001$, respectively). Similarly, patients who were undertriaged to low-acuity ESI levels (4 or 5) on arrival had a significantly increased prevalence of admission and critical outcomes as compared to patients appropriately triaged to the same ESI levels $\left(\chi^{2}=1033.60\right.$, $\mathrm{df}=1, p$ value $<0.001$ and $\chi^{2}=343.05, \mathrm{df}=1, p$ value $<0.001$, respectively).

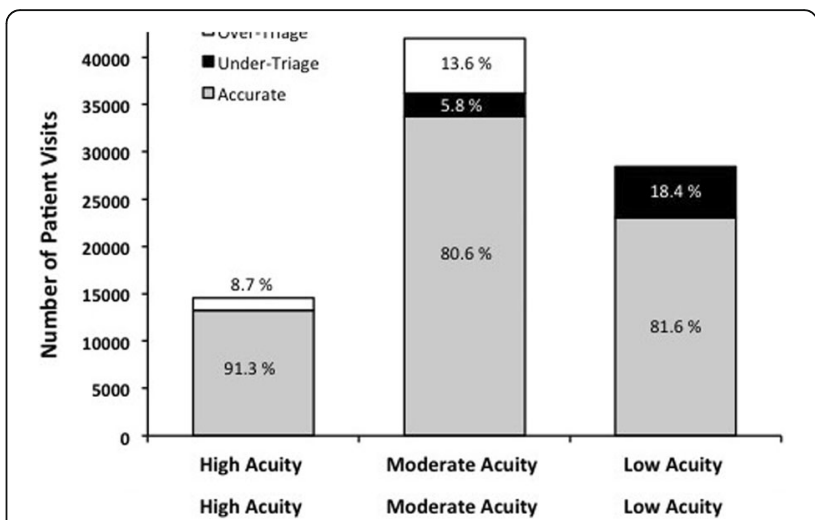

Fig. 1 Distribution of ED visits by initial ESI designation and classifications as accurate, over-triage, or under-triage. High acuity = ESI level 1 or 2; moderate acuity = ESI level 3; low acuity = ESI level 4 or 5. ED emergency department; ESI Emergency Severity Index

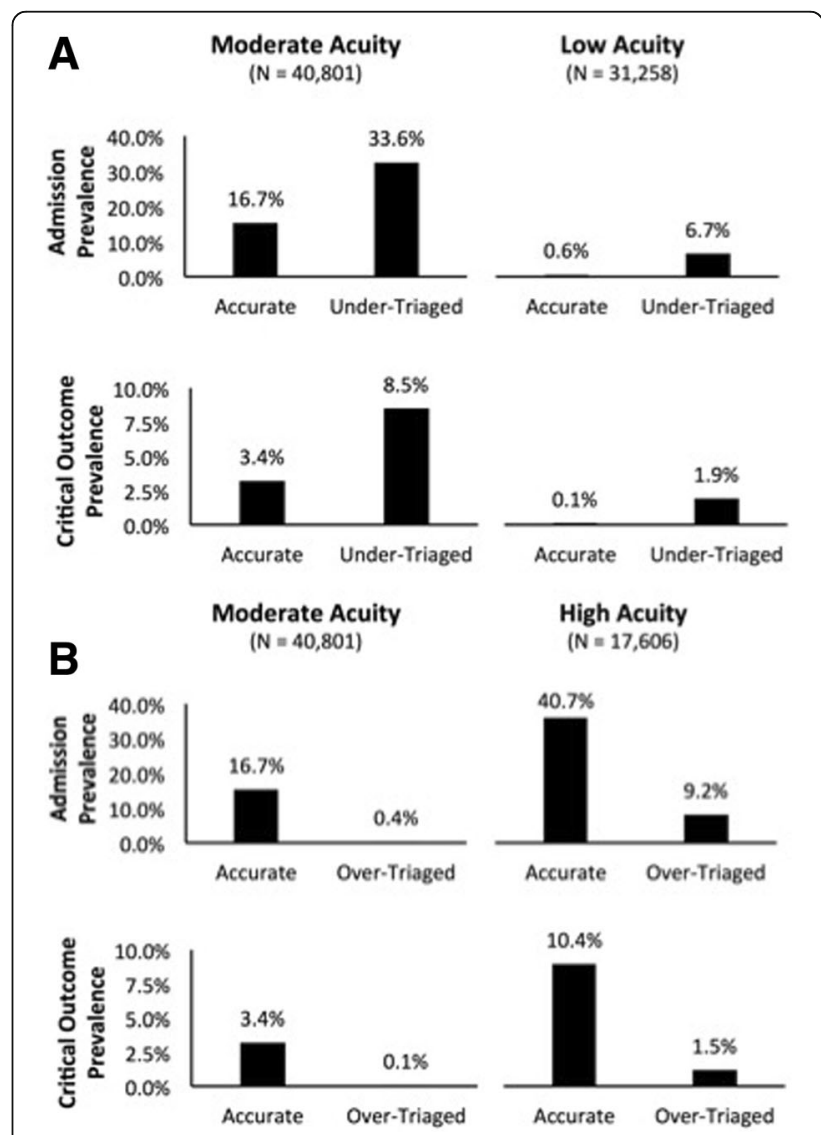

Fig. 2 Associations between triage accuracy and clinical outcomes. a Under-triage to moderate- and low-acuity ESI triage levels is associated with a significantly increased prevalence of hospital admission and critical outcomes. $\mathbf{b}$ Over-triage to moderate- and high-acuity ESI triage levels is associated with a significantly decreased prevalence of hospital admission and critical outcomes 


\section{Over-triage and risk for admission or critical outcome}

The opposite trend was observed for patients who were over-triaged (Fig. 2b). Those triaged as moderate acuity by ESI on arrival but subsequently determined to be more appropriate for low-acuity triage level 4 or 5 (i.e., over-triaged) had a significantly lower prevalence of admission and critical outcomes as compared to those appropriately triaged to moderate acuity $\left(\chi^{2}=1184.90 \mathrm{df}\right.$ $=1, p$ value $<0.001$ and $\chi^{2}=213.04, \mathrm{df}=1, p$ value $<0.001$, respectively). Similarly, patients who were over-triaged to high-acuity ESI levels (1 or 2) on arrival had a significantly lower prevalence of admission and critical outcomes as compared to patients appropriately triaged to the same ESI levels $\left(\chi^{2}=588.49, \mathrm{df}=1, p\right.$ value $<0.001$ and $\chi^{2}=$ $126.57, \mathrm{df}=1, p$ value $<0.001$, respectively).

\section{Factors associated with over-triage and under-triage Age}

Advancing age was associated with under-triage by ESI (Table 2). Odds of under-triage increased in parallel with age and were greatest for patients aged $\geq 70$ years (OR 1.50, 95\% CI 1.30-1.74 for under-triage to moderate acuity designations and OR 2.2, 95\% CI 1.24-3.75 for under-triage to low acuity designations). Conversely, advanced age was associated with low likelihood of overtriage to high or moderate ESI acuity levels (Table 3).

\section{Vital signs}

There were several associations between triage vital signs and under-triage using ESI. Most notably, severe bradycardia, tachycardia, and hypoxia were all strongly associated with under-triage of high-acuity patients to moderate-acuity ESI triage levels (OR 2.55, 95\% CI 1.54-4.02; OR 2.17, 95\% CI 1.61-2.88; and OR 2.18, 95\% CI 1.48-3.12, respectively). Importantly, the absence of oxygen saturation measurements was also strongly associated with under-triage to moderate acuity (OR 2.81, 95\% CI 1.23-5.74). Mild abnormalities in vital signs were more likely to be associated with under-triage to lowacuity ESI levels, including mild tachycardia (OR 1.20, 95\% CI 1.05-1.37) and mild hypotension (OR 1.15, 95\% CI 1.03-1.28). An exception was temperature, where patients with both borderline and frank hyperthermia were both more likely to be under-triaged to low-acuity ESI triage levels (OR 1.49, 95\% CI 1.33-1.66 and OR 1.65, 95\% CI 1.42-1.90, respectively). There were no vital sign abnormalities associated with increased likelihood of over-triage to any acuity level (Table 3 ).

\section{Chief complaints}

A number of chief complaints were associated with under-triage (Table 3). Those most likely to result in a triage of high-acuity patients to moderate-acuity ESI levels included neurologic complaints (OR 1.48, 95\% CI
1.17-1.87), syncope (OR 1.41, 95\% CI 1.03-1.90), and chest pain (OR 1.43, 95\% CI 1.17-1.73). Complaints most likely to be under-triaged to low-acuity ESI levels were chest pain (OR 1.88, 95\% CI 1.54-2.29) and abdominal pain $(1.80,95 \%$ CI 1.57-2.06), followed by shortness of breath (OR 1.49, 95\% CI 1.25-1.78) and back pain (OR 1.47, 95\% CI 1.19-1.82). On the converse, strongest associations with over-triage of low-acuity patients to moderate-acuity triage levels were those related to upper respiratory tract infections (OR 2.82, 95\% CI 2.27-3.50); ear, nose, and throat (ENT) complaints (OR 2.67, 95\% CI 1.99-3.55); skin, nails, and hair complaints (OR 2.32, 95\% CI 1.88-2.87); allergic complaints (OR 1.96, 95\% CI 1.57-2.45); and hypertension (OR 1.88, 95\% CI 1.29-2.66). Lower respiratory tract infections, allergic complaints, and hypertension were most strongly associated with over-triage to high-acuity ESI levels (OR 2.15, 95\% CI 1.28-3.49; OR 2.14, 95\% CI 1.33-3.34; and OR 2.00, 95\% CI 1.02-3.67, respectively).

\section{Discussion}

In this study, we evaluated the predictive accuracy of ED triage by nurses applying ESI using a novel approach that leveraged both clinical judgment of a treating physician with knowledge of actual ED resource utilization and clinical outcome data captured from the EHR. We found that nearly one in five patients was under- or over-triaged by ESI on ED arrival and that this was caused by both under-recognition of high acuity clinical presentations and overestimation of urgency in patients without severe illness. Importantly, we found that under-triage was associated with increased hospital admission rates and greater likelihood for critical clinical outcomes. These findings are consistent with prior findings that failure to distinguish patients with critical and time-sensitive conditions contributes to the delays in disposition and time-sensitive treatments and to the increases in potentially avoidable morbidity and mortality [6-9]. Partly as a result of mistriage, approximately one half of all patients in this population were designated with the same triage score [3] using this five-level index. A similar lack of patient differentiation has also been observed widely in the USA and has been cited as a specific limitation of ESI [24, 30].

Perhaps most importantly, this study identified many factors highly predictive of under- and over-triage by ESI, several of which related to objective data routinely collected on ED arrival. Advanced age was strongly associated with under-triage, suggesting the impact of patient age on initial presentation and clinical course is not well-recognized by ESI and under-appreciated by triage clinicians. While the ESI training manual suggests consideration of age when determining whether a particular presentation is a high risk, no specific recommendations 
Table 2 Factors predictive of under-triage to moderate and low ESI acuity levels ${ }^{\dagger}$

\begin{tabular}{lll}
\hline Moderate acuity & Low acuity \\
(ESI 3) & (ESI 4 or 5) \\
$N=40,801$ & $N=31,258$ \\
& Odds ratio & Odds ratio \\
$(95 \% \mathrm{Cl})$ & $(95 \% \mathrm{Cl})$ \\
\hline
\end{tabular}

Age (18-29 years comparator)

$\begin{array}{lll}30-49 \text { years } & 1.25(1.11-1.41)^{* * *} & 1.15(1.08-1.22)^{* *} \\ 50-69 \text { years } & 1.48(1.3-1.69)^{* * *} & 1.48(1.33-1.63)^{* *} \\ \geq 70 \text { years } & 1.50(1.3-1.74)^{* * *} & 2.20(1.24-3.75)^{*}\end{array}$

Sex (male comparator)

Female

$0.81(0.75-0.88)^{* * *} \quad 1.01(0.95-1.08)$

Null (missing)

$0.21(0.01-0.95)$

$0.02(0.00-0.07)^{* *}$

Temperature (normal comparator)

Hypothermia ( $\leq 94.0^{\circ} \mathrm{F}$ )
Mild hypothermia $\left(94.1-96.2^{\circ} \mathrm{F}\right)$
Mild hyperthermia $\left(99.3-100.4^{\circ} \mathrm{F}\right)$
Hyperthermic ( $\left.\geq 100.5^{\circ} \mathrm{F}\right)$
Null (missing)
Heart rate (normal comparator)
Severe bradycardia ( $\leq 49 \mathrm{bpm})$
Mild bradycardia (50-59 bpm)
Mild tachycardia (105-109 bpm)
Moderate tachycardia
(110-119 bpm)
High tachycardia
(120-129 bpm)
Severe tachycardia
( $\geq 130$ bpm)
Null (missing)

$1.15(0.95-1.39)$

$1.01(0.9-1.13)$

$0.90(0.73-1.08)$

$0.98(0.77-1.24)$

$1.41(1.00-1.94)^{*}$

$2.55(1.54-4.02)^{* * *}$

$1.22(0.99-1.50)$

$1.08(0.88-1.32)$

$1.13(0.94-1.34)$

$1.32(1.01-1.7)^{*}$

$2.17(1.61-2.88)^{* * *}$

$-$
Table 2 Factors predictive of under-triage to moderate and low ESI acuity levels ${ }^{\dagger}$ (Continued)

\begin{tabular}{|c|c|c|}
\hline & $\begin{array}{l}\text { Moderate acuity } \\
\text { (ESI 3) }\end{array}$ & $\begin{array}{l}\text { Low acuity } \\
\text { (ESI } 4 \text { or } 5 \text { ) }\end{array}$ \\
\hline & $N=40,801$ & $N=31,258$ \\
\hline & $\begin{array}{l}\text { Odds ratio }{ }^{\ddagger} \\
(95 \% \mathrm{Cl})\end{array}$ & $\begin{array}{l}\text { Odds ratio } \\
(95 \% \mathrm{Cl})\end{array}$ \\
\hline Severe $[8-10]$ & $1.23(1.11-1.36)^{* * *}$ & $1.11(1.01-1.22)$ \\
\hline Null (missing or $>10$ ) & $0.47(0.17-1.04)$ & $0.42(0.11-1.17)$ \\
\hline \multicolumn{3}{|l|}{ Chief complaint (general comparator) } \\
\hline Abdominal pain & $1.13(0.97-1.31)$ & $1.8(1.57-2.06)^{* *}$ \\
\hline Allergic & $0.49(0.27-0.81)^{*}$ & $0.42(0.30-0.57)^{* *}$ \\
\hline Altered mental status & $1.55(0.98-2.36)$ & $1.74(0.79-3.56)$ \\
\hline Back pain & $1.01(0.81-1.26)$ & $1.47(1.19-1.82)^{* *}$ \\
\hline Chest pain & $1.43(1.17-1.73)^{* * *}$ & $1.88(1.54-2.29)^{* *}$ \\
\hline Dizziness & $0.98(0.79-1.22)$ & $0.91(0.74-1.11)$ \\
\hline Dysrhythmia & $1.23(0.79-1.84)$ & $1.22(0.65-2.14)$ \\
\hline Edema & $0.44(0.29-0.64)^{* * *}$ & $0.71(0.54-0.93)$ \\
\hline Ear, nose, and throat & $0.88(0.47-1.50)$ & $0.27(0.18-0.38)^{* *}$ \\
\hline Fever & $0.61(0.48-0.77)^{* * *}$ & $0.83(0.72-0.95)^{*}$ \\
\hline Genitourinary & $0.69(0.52-0.9)^{* *}$ & $0.95(0.79-1.14)$ \\
\hline Gastrointestinal bleeding & $0.68(0.36-1.19)$ & $0.73(0.31-1.48)$ \\
\hline Headache & $0.82(0.67-1.01)$ & $0.75(0.63-0.88)^{* *}$ \\
\hline Hypertension & $0.60(0.28-1.13)$ & $0.92(0.46-1.69)$ \\
\hline Lower respiratory tract infection & $0.36(0.26-0.47)^{* * *}$ & $0.76(0.66-0.88)^{* *}$ \\
\hline Musculoskeletal (atraumatic) & $0.48(0.27-0.79)^{* *}$ & $0.31(0.22-0.43)^{* *}$ \\
\hline Musculoskeletal (isolated trauma) & $0.39(0.12-0.94)$ & $0.36(0.09-1.03)$ \\
\hline Neurologic & $1.48(1.17-1.87)^{* * *}$ & $1.18(0.82-1.67)$ \\
\hline Nausea, vomiting, and diarrhea & $0.57(0.44-0.73)^{* * *}$ & $0.84(0.71-0.99)$ \\
\hline Seizure & $0.78(0.47-1.22)$ & $1.65(1.08-2.48)$ \\
\hline Shortness of breath & $1.06(0.85-1.32)$ & $1.49(1.25-1.78)^{* *}$ \\
\hline Skin, nails, and hair & $0.30(0.15-0.55)^{* * *}$ & $0.28(0.2-0.38)^{* *}$ \\
\hline Substance abuse & $0.81(0.24-1.99)$ & $0.59(0.14-1.76)$ \\
\hline Syncope & $1.41(1.03-1.90)^{*}$ & $1.68(0.96-2.84)$ \\
\hline Trauma & $0.77(0.59-1.00)$ & $0.70(0.52-0.92)$ \\
\hline Upper respiratory tract infection & $0.44(0.24-0.75)^{* *}$ & $0.26(0.21-0.32)^{* *}$ \\
\hline Weakness & $0.78(0.57-1.05)$ & $0.96(0.76-1.21)$ \\
\hline Wound & $0.35(0.16-0.66)^{* *}$ & $0.45(0.28-0.68)^{* *}$ \\
\hline Other & $0.58(0.39-0.84)^{* *}$ & $0.51(0.38-0.67)^{* *}$ \\
\hline Null (missing) & - & $0.68(0.57-0.81)^{* *}$ \\
\hline \multicolumn{3}{|l|}{ Arrival time (17:00-20:59 comparator) } \\
\hline 01:00-07:59 & $1.23(1.06-1.44)^{* *}$ & $1.77(1.58-1.99)^{* *}$ \\
\hline $08: 00-12: 59$ & $1.48(1.32-1.67)^{* * *}$ & $1.75(1.60-1.90)^{* *}$ \\
\hline 13:00-16:59 & $1.20(1.06-1.36)^{* *}$ & $1.37(1.24-1.50)^{* *}$ \\
\hline 21:00-00:59 & $0.91(0.78-1.06)$ & $1.04(0.92-1.17)$ \\
\hline
\end{tabular}

${ }^{\dagger}$ Results are odds ratios with $95 \%$ confidence intervals in parentheses

bpm beats per minute, rpm respirations per minute, VAS visual analog scale ¥Odds ratio of being assigned ESI triage level 3 on arrival and subsequently being determined more appropriate for ESI level 1 or 2

${ }^{\S}$ Odds ratio of being assigned ESI triage level 4 or 5 on arrival and subsequently

being determined more appropriate for ESI level 1, 2, or 3

*Significant at the $5 \%$ level

**Significant at the $1 \%$ level

***Significant at the $0.1 \%$ level

Pain score (VAS, moderate comparator)

Mild (0-3)

$0.81(0.73-0.9)^{* * *}$

$0.83(0.77-0.88)^{* *}$ 
Table 3 Factors predictive of over-triage to high and moderate ESI acuity levels ${ }^{\dagger}$

\begin{tabular}{|c|c|c|c|c|c|}
\hline \multicolumn{3}{|l|}{16} & \multicolumn{3}{|l|}{ Esl aculty levels (continuea) } \\
\hline & $\begin{array}{l}\text { High acuity } \\
\text { (ESI } 1 \text { or } 2 \text { ) }\end{array}$ & $\begin{array}{l}\text { Moderate acuity } \\
\text { (ESI 3) }\end{array}$ & & $\begin{array}{l}\text { High acuity } \\
\text { (ESI } 1 \text { or } 2 \text { ) }\end{array}$ & $\begin{array}{l}\text { Moderate acuity } \\
\text { (ESI 3) }\end{array}$ \\
\hline & $N=17,606$ & $N=44,470$ & & $N=17,606$ & $N=44,470$ \\
\hline & $\begin{array}{l}\text { Odds ratio } \\
(95 \% \mathrm{Cl})\end{array}$ & $\begin{array}{l}\text { Odds ratio } \\
(95 \% \mathrm{Cl})\end{array}$ & & $\begin{array}{l}\text { Odds ratio }{ }^{\ddagger} \\
(95 \% \mathrm{Cl})\end{array}$ & $\begin{array}{l}\text { Odds ratio } \\
(95 \% \mathrm{Cl})\end{array}$ \\
\hline \multicolumn{3}{|l|}{ Age (18-29 years comparator) } & \multicolumn{3}{|l|}{ Chief complaint (general comparator) } \\
\hline 30-49 years & $0.80(0.68,0.93)^{* *}$ & $0.79(0.73,0.84)^{* * *}$ & Abdominal pain & $0.84(0.67,1.06)$ & $0.77(0.69-0.87)^{* * *}$ \\
\hline 50-69 years & $0.62(0.52,0.74)^{* * *}$ & $0.65(0.6-0.71)^{* * *}$ & Allergic & $2.14(1.33,3.34)^{* *}$ & $1.96(1.57-2.45)^{* * *}$ \\
\hline$\geq 70$ years & $0.37(0.31,0.46)^{* * *}$ & $0.42(0.37-0.46)^{* * *}$ & Altered mental status & $0.63(0.30,1.17)$ & $0.36(0.16-0.69)^{* *}$ \\
\hline \multicolumn{3}{|l|}{ Sex (male comparator) } & Back pain & $0.83(0.61,1.11)$ & $1.08(0.91-1.27)$ \\
\hline Female & $1.40(1.24-1.57)^{* * *}$ & $1.12(1.06-1.19)^{* * *}$ & Chest pain & $1.04(0.78,1.4)$ & $0.68(0.57-0.81)^{* * *}$ \\
\hline Null (missing) & - & $0.68(0.26-1.49)$ & Dizziness & $1.36(0.97,1.89)$ & $1.13(0.96-1.32)$ \\
\hline \multicolumn{3}{|l|}{ Temperature (normal comparator) } & Dysrhythmia & $1.31(0.72,2.24)$ & $1.00(0.72-1.38)$ \\
\hline Hypothermia $\left(\leq 94.0^{\circ} \mathrm{F}\right)$ & $0.80(0.63,1.01)$ & $1.05(0.97-1.14)$ & Edema & $1.33(0.7,2.33)$ & $1.37(1.12-1.67)^{* *}$ \\
\hline Mild hypothermia $\left(94.1-96.2^{\circ} \mathrm{F}\right)$ & $1.00(0.86,1.17)$ & $1.03(0.89-1.19)$ & Ear, nose, and throat & $1.67(0.88,2.97)$ & $2.67(1.99-3.55)^{* * *}$ \\
\hline Mild hyperthermia (99.3-100.4 º $\mathrm{F})$ & $0.99(0.73,1.33)$ & $0.81(0.71-0.92)^{* *}$ & Fever & $1.52(1.01,2.25)^{*}$ & $0.94(0.81-1.10)$ \\
\hline Hyperthermic ( $\geq 100.5^{\circ} \mathrm{F}$ ) & $1.28(0.89,1.79)$ & $0.66(0.54-0.79)^{* * *}$ & Genitourinary & $1.23(0.81,1.81)$ & $1.08(0.90-1.28)$ \\
\hline Null (missing) & $0.68(0.40,1.09)$ & $0.93(0.71-1.19)$ & Gastrointestinal bleeding & $1.04(0.53,1.88)$ & $1.06(0.73-1.51)$ \\
\hline \multicolumn{3}{|l|}{ Heart rate (normal comparator) } & Headache & $1.21(0.92,1.58)$ & $1.38(1.21-1.58)^{* * *}$ \\
\hline Severe bradycardia ( $\leq 49$ bpm) & $0.46(0.19,0.91)^{*}$ & $0.75(0.39-1.31)$ & Hypertension & $2.00(1.02,3.67)^{*}$ & $1.88(1.29-2.66)^{* * *}$ \\
\hline Mild bradycardia (50-59 bpm) & $1.11(0.83,1.46)$ & $0.99(0.83-1.17)$ & Lower respiratory tract infection & $2.15(1.28,3.49)^{* *}$ & $1.43(1.23-1.66)^{* * *}$ \\
\hline Mild tachycardia (105-109 bpm) & $0.83(0.61,1.11)$ & $0.89(0.78-1.03)$ & Musculoskeletal (atraumatic) & $1.62(0.83,2.92)$ & $2.21(1.76-2.77)^{* * *}$ \\
\hline Moderate tachycardia (110-119 bpm) & $0.84(0.66,1.05)$ & $0.97(0.86-1.09)$ & Musculoskeletal (isolated trauma) & $1.02(0.24,2.91)$ & $0.70(0.36-1.24)$ \\
\hline High tachycardia (120-129 bpm) & $0.83(0.61,1.11)$ & $0.94(0.78-1.13)$ & Neurologic & $1.13(0.84,1.52)$ & $0.79(0.64-0.96)^{*}$ \\
\hline Severe tachycardia ( $\geq 130 \mathrm{bpm}$ ) & $0.35(0.23,0.51)^{* * *}$ & $0.88(0.67-1.14)$ & Nausea, vomiting, and diarrhea & $1.37(0.99,1.88)$ & $1.33(1.15-1.54)^{* * *}$ \\
\hline Null (missing) & $0.49(0.05,4.48)$ & $1.23(0.22-6.74)$ & Seizure & $0.85(0.48,1.41)$ & $0.77(0.53-1.08)$ \\
\hline \multicolumn{3}{|l|}{ Systolic blood pressure (normal comparator) } & Shortness of breath & $1.33(0.96,1.82)$ & $0.89(0.75-1.06)$ \\
\hline Hypotension ( $\leq 99$ mmHg) & $0.71(0.55,0.91)^{* *}$ & $0.85(0.73-0.97)^{*}$ & Skin, nails, and hair & $1.65(0.77,3.21)$ & $2.32(1.88-2.87)^{* * *}$ \\
\hline Mild hypotension (100-107 mmHg) & $1.04(0.82,1.3)$ & $0.95(0.86-1.06)$ & Substance abuse & $2.11(0.76,4.97)$ & $0.45(0.14-1.11)$ \\
\hline Mild hypertension (177-199 mmHg) & $0.94(0.75,1.17)$ & $0.78(0.65-0.92)^{* *}$ & Syncope & $0.69(0.45,1.03)$ & $0.75(0.56-0.98)^{*}$ \\
\hline Hypertension ( $\geq 200$ mmHg) & $1.02(0.75,1.38)$ & $0.99(0.63-1.48)$ & Trauma & $0.92(0.65,1.30)$ & $0.89(0.73-1.07)$ \\
\hline Null (missing) & $1.89(1.04,3.22)^{*}$ & - & Upper respiratory tract infection & $2.11(0.70,5.24)$ & $2.82(2.27-3.5)^{* * *}$ \\
\hline \multicolumn{3}{|l|}{ Respiratory rate (normal comparator) } & Weakness & $1.71(1.13,2.54)^{* *}$ & $1.36(1.12-1.65)^{* *}$ \\
\hline Hypopnea ( 13 rpm) & $0.00(0.00,0.01)$ & $1.11(0.56-2.01)$ & Wound & $0.64(0.22,1.48)$ & $1.58(1.2-2.06)^{* * *}$ \\
\hline Mild hypopnea (14-15 rpm) & $0.77(0.57,1.03)$ & $1.04(0.92-1.18)$ & Other & $0.94(0.59,1.44)$ & $1.39(1.14-1.69)^{* *}$ \\
\hline Mild tachypnea (20-22 rpm) & $1.05(0.92,1.19)$ & $1.04(0.97-1.11)$ & Null (missing) & $1.02(0.79,1.31)$ & $1.00(0.87-1.14)$ \\
\hline Moderate tachypnea (23-27 rpm) & $0.96(0.78,1.17)$ & $0.87(0.76-1.00)^{*}$ & \multicolumn{3}{|l|}{ Arrival time (17:00-20:59 comparator) } \\
\hline Severe tachypnea ( $\geq 28$ rpm) & $0.75(0.47,1.14)$ & $0.79(0.49-1.22)$ & 01:00-07:59 & $1.02(0.83,1.25)$ & $0.84(0.76-0.94)^{* *}$ \\
\hline Null (missing) & $2.84(0.35,14.23)$ & $0.80(0.11-4.28)$ & $08: 00-12: 59$ & $1.37(1.17,1.61)^{* * *}$ & $1.23(1.14-1.33)^{* * *}$ \\
\hline \multicolumn{3}{|l|}{ Oxygen saturation (normal comparator) } & 13:00-16:59 & $1.21(1.02,1.43)^{*}$ & $1.07(0.99-1.16)$ \\
\hline Severe hypoxia (SpO2 $\leq 89)$ & $0.40(0.25,0.62)^{* * *}$ & $0.85(0.55-1.25)$ & $21: 00-00: 59$ & $0.99(0.82,1.19)$ & $0.76(0.69-0.84)^{* * *}$ \\
\hline Moderate hypoxia (SpO2 90-94) & $0.72(0.59,0.88)^{* *}$ & $0.62(0.55-0.7)^{* * *}$ & \multirow{7}{*}{\multicolumn{3}{|c|}{ 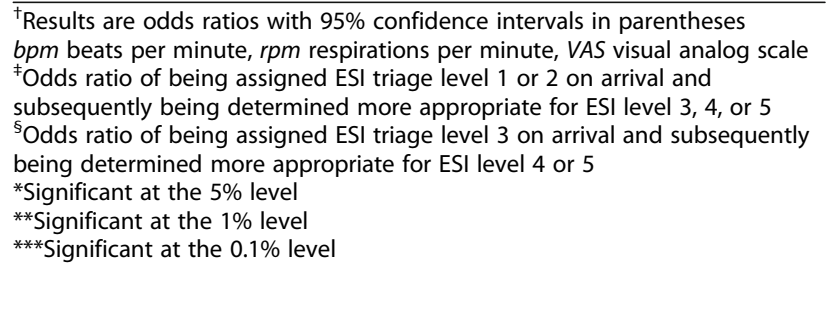 }} \\
\hline Mild hypoxia (SpO2 95-96) & $0.81(0.67,0.97)^{*}$ & $0.86(0.78-0.94)^{* * *}$ & & & \\
\hline Null (missing) & $0.31(0.05,1.2)$ & $0.67(0.21-1.68)$ & & & \\
\hline \multicolumn{3}{|l|}{ Pain score (VAS, moderate comparator) } & & & \\
\hline Mild (0-3) & $0.80(0.68,0.95)^{*}$ & $0.86(0.78-0.94)^{* * *}$ & & & \\
\hline Severe $[8-10]$ & $0.79(0.68,0.93)^{* *}$ & $0.85(0.55-1.25)$ & & & \\
\hline Null (missing or $>10$ ) & $0.23(0.06,0.67)^{*}$ & $0.67(0.21-1.68)$ & & & \\
\hline
\end{tabular}

Table 3 Factors predictive of over-triage to high and moderate ESI acuity levels ${ }^{\dagger}$ (Continued)

rith $95 \%$ confidence intervals in parentheses Odds ratio of being assigned ESI triage level 1 or 2 on arrival and

fently being determined more appropriate for ESI level 3, 4, ing determined more appropriate for ESI level 4 or 5

$0.79(0.68,0.93)^{* *} \quad 0.85(0.55-1.25)$ 
or guidelines related to age are specified [20] and ESI has previously been shown to have poor sensitivity for identification of elderly patients requiring life-saving interventions [31].

Similarly, there was a strong association between multiple vital sign abnormalities (bradycardia, tachycardia, hypotension, hypoxia, and hyperthermia) and undertriage, again suggesting under-appreciation by ESI. It has been well-established that abnormalities in triage vital signs are strong predictors of adverse outcomes including ICU admission and in-hospital mortality [25]. Specific recommendations related to vital signs are provided by ESI, with a suggestion to consider increasing the acuity level assignment for patients with "danger zone vitals," defined as tachycardia (heart rate > $100 \mathrm{bpm}$ ), tachypnea (respiratory rate $>20 \mathrm{rpm}$ ), or hypoxia $(\mathrm{SpO} 2<92 \%)$ [20]. No specific recommendations are provided for patients with bradycardia or derangements in blood pressure or temperature. Good correlation between abnormal vital signs and ESI acuity level assignment was previously reported, but authors of this study utilized an aggregate vital sign score for analysis and the finding that abnormal vital signs are, in general, more likely to fall into higher acuity triage levels should not be surprising [32]. On the converse, our study demonstrates that subtle vital sign abnormalities are systematically under-appreciated by ESI that vital sign derangements for which there are no recommendations provided by ESI are under-recognized and associated with mistriage.

Last, we found strong associations between several chief complaint categories and mistriage. Many of these are understood intuitively. For example, strongest associations with over-triage were seen for allergic reactions, ENT complaints, and respiratory infections (Table 3). Worst-case scenarios for patients in each of these categories involve compromise of airway and breathing. However, the actual severity of illness may not be readily apparent to the triage provider who is without access to a full exam and diagnostics. Under a system that relies heavily on nursing judgment, it is easy to understand how such patients might be triaged to acuity levels higher than ultimately needed. Other associations, however, are more surprising. Chest pain and shortness of breath, for example, are complaints strongly associated with high morbidity conditions including acute coronary syndrome and pulmonary embolism [33, 34], yet both were associated with under-triage in this study. While some standardized triage scales including CTAS [18] and MTS [19] utilize standardized lists of presenting complaints or symptoms in triage score assignment $[18,19]$, they are not addressed directly in the ESI algorithm. Instead, nurses are instructed to consider the presenting complaint in the entire context of the patient when assigning a triage score [20], generating a potential for high variability among users.

While our findings suggest several potential weaknesses of ESI, the scale remains an extremely popular triage tool with many strengths. The simplistic algorithm by which ESI scores are assigned allows nurses to make rapid triage decisions by answering only three questions: (1) Is the patient dying? (2) Should the patient wait? and (3) How many resources will this patient require? [20] While the answers to these questions are certainly influenced by additional factors including patient appearance, chief complaint, and vital signs, a level of autonomy is maintained by the triage provider using ESI that allows for increased efficiency and ease of use. The simplicity of the ESI algorithm is also an important operational strength, as the algorithm can be memorized easily and there is little need for cumbersome paper or electronic reference material in the clinical environment. Finally, the incorporation of resource utilization prediction by ESI is unique among ED triage tools and makes it particularly useful in limited resource settings.

\section{Limitations}

This study is strengthened by its large sample size and the inclusion of an a posteriori triage score assignment with EHR-based clinical outcome validation as comparator, yet several limitations of our findings should be considered. ESI aims to assign patients to triage categories based on both severity of disease presentation and anticipated ED resource utilization. While all physicians who assigned a posteriori ESI scores had full knowledge of actual ED resource utilization, the retrospective nature of our work limits our ability to confirm that these scores accurately reflect resource utilization. However, ED resource utilization has been shown to correlate well with disposition and clinical outcomes, and our findings that patients deemed under-triaged were more likely to be hospitalized or experience critical events supports the validity of our comparator [24, 35, 36]. Additionally, all patient encounters occurred at a single institution where ED nurses undergo structured and ongoing training in ESI triage. It is possible that both distribution of triage score assignment and clinical course at other sites may differ. Indeed, it is likely that in settings where ESI has been adopted for clinical use without implementation of structured training programs for ED nurses, under- and over-triage would be more frequent than observed here and our findings may represent an overestimate of ESI performance in general.

\section{Conclusions}

ESI has many strengths and is among the most widely used of all standardized triage tools, yet a large number of patients in our cohort were under- and over-triaged 
using this scale. Despite rigorous training of triage providers, reliance on human experience and intuition under the ESI algorithm allowed for under-appreciation of the clinical impact of age, subtle vital sign abnormalities, and multiple specific complaints. These findings should be used to inform ESI users, ESI training programs, and future iterations of ESI. Perhaps more importantly, these findings provide a rationale for the development of future triage tools that are both efficient and objective.

\section{Acknowledgements}

We would like to acknowledge the ED nursing and clinical leadership at Hospital Moinhos de Vento and the ED research leadership at Johns Hopkins for their support of this project. In particular, we would also like to thank Ana Costache and Luiza Schlatter Mendoca for the administrative support they provided to facilitate this transcontinental collaboration.

\section{Funding}

This project was supported by a Synergy Award from the Johns Hopkins School of Medicine and grant number R21HS023641 from the Agency for Healthcare Research and Quality. The content is solely the responsibility of the authors and does not necessarily represent the official views of the Agency for Healthcare Research or Johns Hopkins University.

\section{Availability of data and materials}

A limited and de-identified version of the dataset analyzed in this study is available upon written request and upon approval by the institutional review boards of participating institutions

\section{Authors' contributions}

$\mathrm{JH}, \mathrm{SL}, \mathrm{SR}$, and PS conceived and designed this study, and DR and JS assisted in the refinement and presentation of findings. DM and MT performed the data extraction, cleaning, and analysis. JH led the manuscript writing, and all authors contributed to the manuscript revision. All authors read and approved the final manuscript.

\section{Ethics approval and consent to participate}

This study was approved by the institutional review boards of all participating institutions.

\section{Consent for publication}

Not applicable.

\section{Competing interests}

$\mathrm{SL}$ is the founder and co-owner of a start-up company with a focus on datadriven improvement of emergency department and hospital operations. $\mathrm{JH}$, DM, PS, MT, DR, JS, and SR have no competing interests.

\section{Publisher's Note}

Springer Nature remains neutral with regard to jurisdictional claims in published maps and institutional affiliations.

\section{Author details \\ 'Department of Emergency Medicine, Johns Hopkins University School of Medicine, 801 Smith Avenue, Davis Building, Suite 3220, Baltimore, MD 21209, USA. 'Department of Operations Integration, Johns Hopkins Hospital Baltimore, MD, USA. ${ }^{3}$ Emergency Department, Hospital Moinhos de Vento, Porto Alegre, Brazil. ${ }^{4}$ Whiting School of Engineering, Johns Hopkins University, Baltimore, MD, USA. ${ }^{5}$ Systems Institute, Johns Hopkins University, Baltimore, MD, USA.}

Received: 24 April 2017 Accepted: 26 December 2017

Published online: 15 January 2018

\section{References}

1. Iserson KV, Moskop JC. Triage in medicine, part l: concept, history, and types. Ann Emerg Med. 2007;49(3):275-81.
2. Committee on the Future of Emergency Care in the United States Health System. Hospital-based emergency care: at the breaking point. Washington, DC: Committee on the Future of Emergency Care in the United States Health System; 2006

3. Morrison JB, Wears RL. Emergency department crowding: vicious cycles in the ED. In: 29th International Conference of the System Dynamics Society: 2011. p. 1-36.

4. Pines JM, Hilton JA, Weber EJ, Alkemade AJ, Al Shabanah H, Anderson PD, et al. International perspectives on emergency department crowding. Acad Emerg Med. 2011;18(12):1358-70.

5. Stead LG, Jain A, Decker WW. Emergency department over-crowding: a global perspective. Int J Emerg Med. 2009;2(3):133-4.

6. Seiger $N$, van Veen M, Steyerberg EW, Ruige M, van Meurs AHJ, Moll HA. Undertriage in the Manchester triage system: an assessment of severity and options for improvement. Arch Dis Child. 2011;96(7):653-7.

7. Hitchoock M, Gillespie B, Crilly J, Chaboyer W. Triage: an investigation of the process and potential vulnerabilities. J Adv Nurs. 2014;70(7):1532-41.

8. Yurkova I, Wolf L. Under-triage as a significant factor affecting transfer time between the emergency department and the intensive care unit. J Emerg Nurs. 2011;37(5):491-6.

9. Haas B, Gomez D, Zagorski B, Stukel TA, Rubenfeld GD, Nathens AB. Survival of the fittest: the hidden cost of undertriage of major trauma. J Am Coll Surg. 2010;211(6):804-11.

10. Schull MJ, Vermeulen M, Slaughter G, Morrison L, Daly P. Emergency department crowding and thrombolysis delays in acute myocardial infarction. Ann Emerg Med. 2004;44(6):577-85.

11. Kulstad EB, Kelley KM. Overcrowding is associated with delays in percutaneous coronary intervention for acute myocardial infarction. Int J Emerg Med. 2009;2(3):149-54.

12. Pines JM, Hollander JE, Localio AR, Metlay JP. The association between emergency department crowding and hospital performance on antibiotic timing for pneumonia and percutaneous intervention for myocardial infarction. Acad Emerg Med. 2006;13(8):873-8.

13. Sills MR, Fairclough D, Ranade D, Kahn MG. Emergency department crowding is associated with decreased quality of care for children with acute asthma. Ann Emerg Med. 2011:57(3):191-7.

14. Mills AM, Shofer FS, Chen EH, Hollander JE, Pines JM. The association between emergency department crowding and analgesia administration in acute abdominal pain patients. Acad Emerg Med. 2009;16(7):603-8.

15. Oredsson $\mathrm{S}$, Jonsson $H$, Rognes J, Lind L, Goransson KE, Ehrenberg A, et al. A systematic review of triage-related interventions to improve patient flow in emergency departments. Scand J Trauma Resusc Emerg Med. 2011;19:43.

16. Calder LA, Forster AJ, Stiell IG, Carr LK, Perry JJ, Vaillancourt C, et al. Mapping out the emergency department disposition decision for high-acuity patients. Ann Emerg Med. 2012;60(5):567-576.e4.

17. Australasian College for Emergency Medicine. Guidelines on the implementation of the Australasian Triage Scale in Emergency Department. Doc No G24. 2013;1-8.

18. Beveridge R, Clarke B, Janes L, Savage N, Thompson J, Dodd G, et al. Canadian Emergency Department Triage and Acuity Scale: implementation guidelines. Can J Emerg Med. 1999;1(3 suppl):S2-28.

19. Mackway-Jones K, editor. Emergency triage. 1st ed. Manchester: Manchester Triage Group; 1996. p. 168.

20. Ahrq Q. In: Gilboy N, Tanabe P, Travers D, Rosenau AM, editors. Emergency Severity Index (ESI): a triage tool for emergency department care. 4th ed; 2012. p. 114.

21. McHugh M, Tanabe P, McClelland M, Khare RK. More patients are triaged using the Emergency Severity Index than any other triage acuity system in the United States. Acad Emerg Med. 2012;19(1):106-9.

22. Christ M, Grossmann F, Winter D, Bingisser R, Platz E. Modern triage in the emergency department. Dtsch Arztebl Int. 2010;107(50):892-8.

23. Center for Disease Control and Prevention. Ambulatory Health Care Data. [lnternet]. [cited 2014 Jan 1]. Available from: http://www.cdc.gov/nchs/ahcd.htm

24. Dugas AF, Kirsch TD, Toerper M, Korley F, Yenokyan G, France D, et al. An electronic emergency triage system to improve patient distribution by critical outcomes. J Emerg Med. 2016;50(6):910-8.

25. Barfod C, Lauritzen MMP, Danker JK, Sölétormos G, Forberg JL, Berlac PA, et al. Abnormal vital signs are strong predictors for intensive care unit admission and in-hospital mortality in adults triaged in the emergency department-a prospective cohort study. Scand I Trauma Resusc Emerg Med. 2012;20:28 
26. Tortora GJ. Principles of Anatomy and Physiology. 6th ed. Boston: AddisonWesley Educational Publishers; 1990. 956.

27. Chobanian AV, Bakris GL, Black HR, et al. The seventh report of the joint national committee on prevention, detection, evaluation, and treatment of high blood pressure: the jnc 7 report. JAMA. 2003;289(19):2560-71.

28. McGillicuddy DC, O'Connell FJ, Shapiro NI, Calder SA, Mottley LJ, Roberts JC, et al. Emergency department abnormal vital sign "triggers" program improves time to therapy. Acad Emerg Med. 2011;18(5):483-7.

29. Healthcare cost and utilization project: clinical classification software [Internet]. Agency for Healthcare Research in Quality. [cited 2016 Jan 1]. Available from: http://www.hcup-us.ahrq.gov/toolssoftware/ccs/ccs.jsp

30. Centers for Disease Control and Prevention. National Hospital Ambulatory Medical Care Survey: 2010 emergency department summary tables. 2011.

31. Platts-Mills TF, Travers D, Biese K, McCall B, Kizer S, LaMantia M, et al. Accuracy of the emergency severity index triage instrument for identifying elder emergency department patients receiving an immediate life-saving intervention. Acad Emerg Med. 2010;17(3):238-43.

32. van der Wulp I, Rullmann HA, Leenen LP, van Stel HF. Associations of the Emergency Severity Index triage categories with patients' vital signs at triage: a prospective observational study. Emerg Med J. 2011;28(12):1032-5.

33. Devos E, Jacobson L. Approach to adult patients with acute dyspnea. Emerg Med Clin North Am. 2016:34(1):129-49.

34. Woo KC, Schneider Jl. High-risk chief complaints I: chest pain —-the big three. Emerg Med Clin North Am. 2009;27(4):685-712.

35. Eitel DR, Travers DA, Rosenau AM, Gilboy N, Wuerz RC. The emergency severity index triage algorithm version 2 is reliable and valid. Acad Emerg Med. 2003;10(10):1070-80.

36. Wuerz RC, Milne LW, Eitel DR, Travers D, Gilboy N. Reliability and validity of a new five-level triage instrument. Acad Emerg Med. 2000;7(3):236-42.

\section{Submit your manuscript to a SpringerOpen ${ }^{\circ}$ journal and benefit from:}

- Convenient online submission

Rigorous peer review

- Open access: articles freely available online

- High visibility within the field

- Retaining the copyright to your article

Submit your next manuscript at $\gg$ springeropen.com 